\title{
Experimental investigations on lasers FM and AM noise
}

\author{
Olivier Llopis, Gilles Bailly, Alexis Bougaud, Arnaud Fernandez \\ LAAS-CNRS, Université de Toulouse, CNRS, UPS, Toulouse, France \\ Contact author: 1lopis@laas.fr
}

\begin{abstract}
Different $1550 \mathrm{~nm}$ fibered lasers are compared versus their performances in terms of frequency (FM) noise and amplitude (AM) noise. The noise spectral density is spread over six to height decades for the FM noise and three to four decades for the AM noise, depending on the frequency offset. The lasers featuring the best FM noise performance are generally the worst versus the AM noise performance, although some compromises can be found.
\end{abstract}

Keywords-laser, semiconductor laser, fiber laser, frequency noise, phase noise, amplitude noise, noise characterization

\section{INTRODUCTION}

Laser frequency (FM) noise is the limiting parameter for many applications in time \& frequency domain, but also in optical interferometric sensors and LIDARs. This noise may also be converted into RF phase noise in a long distance optical link through the fiber dispersion.

Laser amplitude (AM) noise is the signal to noise limiting parameter in a short length optical link. The laser $1 / \mathrm{f}$ $\mathrm{AM}$ noise is also converted into RF phase noise at the photodiode level and is an important problem in low RF phase noise optical links or optoelectronic oscillators design.

Laser tunability bandwidth is another important parameter. It is very large for a free running semiconductor laser $(\Delta v>500 \mathrm{GHz})$ but it may be significantly reduced when the laser is stabilized on an external resonator.

An ideal optical source would be a laser featuring simultaneously low FM noise, low AM noise and wide tunable bandwidth. Of course, such an optical source does not exists and some compromises must be made between all these parameters.

We present and discuss in this paper a set of experimental results obtained on two semiconductor DFB lasers, two external cavity stabilized semiconductor lasers, a laboratory fiber laser and an in-house laboratory laser stabilized on an ultra-high Q resonator.

\section{MEASUREMENT SET-UP}

The lasers FM noise spectrum is measured using a $2 \mathrm{~km}$ delay line self-heterodyne frequency discriminator [1,2]. The system performance has been presented at IFCS 2015 [3]. Since this presentation, it has been improved by replacing the $80 \mathrm{MHz}$ intermediate frequency oscillator by a low phase noise synthesizer, the IF oscillator noise being the limiting parameter at very low offset frequencies $(1 \mathrm{~Hz}$ to $5 \mathrm{~Hz})$. It has also been improved by protecting the whole system from mechanical and acoustic vibrations, which are still the limiting factor in the $10 \mathrm{~Hz}$ to $1 \mathrm{kHz}$ range. This system is shown in Figure 1.

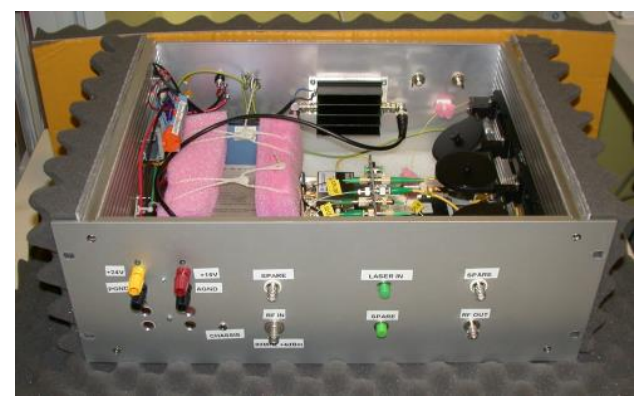

Fig. 1. Optical FM noise measurement system: $2 \mathrm{~km}$ delay line frequency discriminator with $80 \mathrm{MHz}$ frequency offset obtained with an acousto-optic modulator. The output signal at $80 \mathrm{MHz}$ is measured on a E5052B RF phase noise analyzer.

The laser AM noise has been measured directly on a high power photodiode with a baseband FFT analyzer and a low noise amplifier. An optical attenuator is added to prevent the photodiode saturation when the optical power received on the photodiode exceeds $10 \mathrm{~mW}$. The measured optical AM noise is referenced to the detected laser power and thus plot as relative intensity noise (RIN).

\section{LASERS UNDER TEST}

Five commercial $1550 \mathrm{~nm}$ lasers have been measured. Lasers 1 and 2 are two semiconductor DFB lasers. Laser 1 is a $20 \mathrm{~mW}$ grade laser and corresponds to an old technology (supplied more than 10 years ago). Laser 2 is a $40 \mathrm{~mW}$ grade laser recently supplied. Laser 3 is a tunable laser module from IDPhotonics, based on an external cavity with a piezoelectric controlled end mirror and temperature controlled filters. Laser 4 is a fixed frequency external cavity DFB laser module from RIO (RIO PLANEX ${ }^{\mathrm{TM}}$ ). Laser 5 is a laboratory fiber laser from Koheras (today NKT Photonics) available in a rack size benchtop.

To these five commercial devices, we have add for comparison the frequency noise measurement of an homemade optical source [4] which is a RIO laser stabilized on a ultra-high $\mathrm{Q}$ factor fiber ring resonator $\left(\mathrm{Q}=210^{9}\right)$ with a Pound Drever Hall (PDH) system. The PDH system is based on a low noise three stage PID and is optimized for the small free spectral range of the fiber ring resonator $(2 \mathrm{MHz})$. The resonator includes an intra-cavity isolator to prevent the start-up of some nonlinear effects such as stimulated Brillouin scattering.

Table 1 depicts the tunability properties of these sources. Only the free running DFB lasers 1 and 2 and the IDPhotonics source are tunable on large frequency bandwidths, but the IDPhotonics source is not continuously tunable. 


\begin{tabular}{|c|c|c|}
\hline & $\begin{array}{c}\text { Fast tuning } \\
\text { continuous FM coeff. }\end{array}$ & $\begin{array}{c}\text { Slow tuning } \\
\text { with temperature }\end{array}$ \\
\hline 1) DFB $1-20 \mathrm{~mW}$ & $1100 \mathrm{MHz} / \mathrm{mA}$ & $>500 \mathrm{GHz}$ \\
\hline 2) DFB $2-40 \mathrm{~mW}$ & $600 \mathrm{MHz} / \mathrm{mA}$ & $>500 \mathrm{GHz}$ \\
\hline 3) IDPhotonics & $40 \mathrm{MHz} / \mathrm{V}$ & $7500 \mathrm{GHz}$ \\
\hline 4) RIO Planex & $100 \mathrm{MHz} / \mathrm{mA}$ & $\sim 30 \mathrm{GHz}$ \\
\hline 5) Koheras Adjustik & $15 \mathrm{MHz} / \mathrm{V}$ & $125 \mathrm{GHz}$ \\
\hline
\end{tabular}

Table 1: lasers frequency tunability

\section{FREQUENCY NOISE MEASUREMENTS}

The results of the frequency noise measurements are depicted in Figure 2. As expected, the two free running DFB lasers are the noisiest ones. However, laser 2 performance is relatively close to the performance of the external cavity stabilized DFB lasers (lasers 3 and 4). From the frequency noise floor far from the carrier $(100 \mathrm{kHz})$, it is possible to compute the instantaneous linewidth $(\tau<10 \mu$ s) of these DFB lasers by multiplying the minimum frequency noise spectral density by $\pi$ (white frequency noise laser model). Laser 1 instantaneous linewidth is close to $1 \mathrm{MHz}$ while laser 2 linewidth is about $100 \mathrm{kHz}$.

In the middle range in terms of frequency noise performances, we found the external cavity semiconductor lasers (IDPhotonics and RIO).

The two best lasers are the fiber lasers, with the Koheras laser first and finally, the quietest one, the RIO laser stabilized on fiber ring resonator. This last laser features a $30 \mathrm{~Hz}$ linewidth for $5 \mathrm{~ms}$ integration time [4]. However, the measured frequency noise for this laser is very close to the measurement system noise floor and is limited by the reference spool vibrations between $10 \mathrm{~Hz}$ and $1 \mathrm{kHz}$ offset [3]. In this frequency offset range, laser 6 performance may be better than the one measured and depicted in Figure 2.

Finally, the differences in performances are strong and largely determined by the differences in resonator Q factor. As expected, large resonators with high $\mathrm{Q}$ factor results in high spectral purity sources.

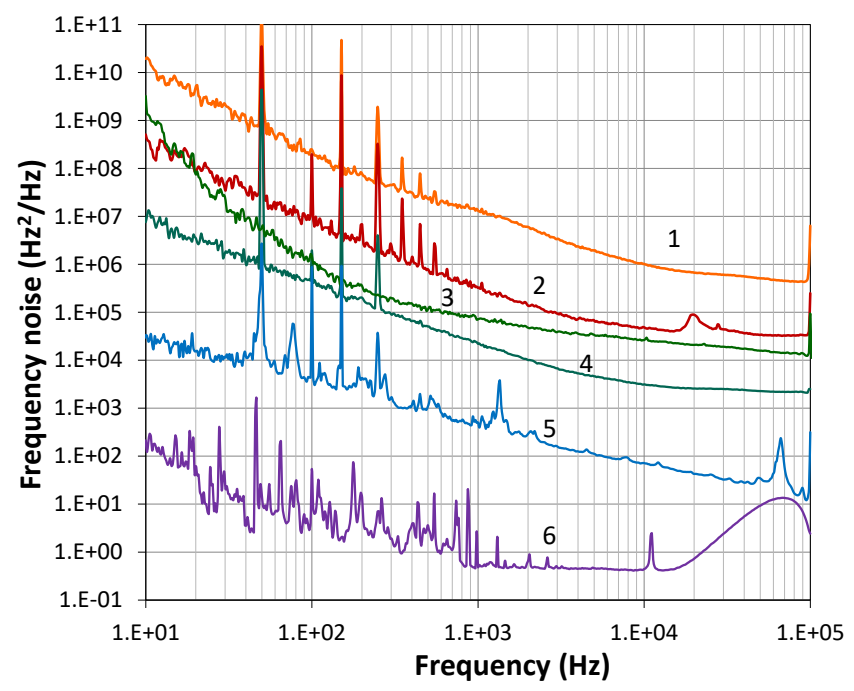

Figure 2: Frequency noise spectral density of six different sources (four commercially available and one home-made). $20 \mathrm{~mW}$ (orange: 1) and $40 \mathrm{~mW}$ (red: 2) DBFs, IDPhotonics tunable laser (green: 3), RIO semiconductor external cavity laser - free running (blue-green: 4), Koheras fiber laser (blue: 5), RIO laser stabilized on a $Q=210^{9}$ fiber ring resonator with a PDH control loop (purple: 6 )

\section{AMPLITUDE NOISE MEASUREMENTS}

The results of the AM noise measurements are depicted in Figure 3, at least for laser 1 to 5. The lasers classification is almost the opposite with the fiber laser being the noisier one close to the carrier, then the tunable external cavity laser, then the fixed frequency external cavity laser and then the two DFB lasers. Laser 4 represents however a good compromise to get good FM noise and AM noise performance simultaneously.

Close to the carrier, most of these sources feature a close to $1 / \mathrm{f}$ amplitude noise, with the exception of the fiber laser 5: the bump on the spectrum of this laser is typical of slow relaxation noise (or generation-recombination noise). The $1 / \mathrm{f}$ AM noise of the other lasers spreads on three decades in amplitude, revealing strong differences in semiconductor devices quality. Laser 2 (40 mW DFB) is the best device in terms of AM noise, with an extremely low $1 / \mathrm{f}$ noise corner frequency $(4 \mathrm{kHz})$ and a low white $\mathrm{AM}$ noise floor measured at $-163 \mathrm{dBc} / \mathrm{Hz}$.

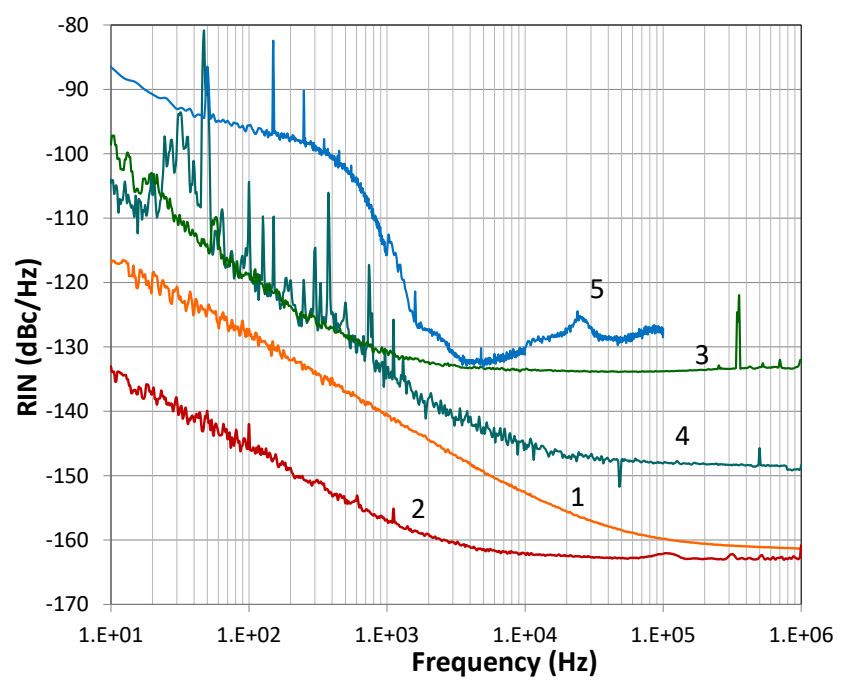

Figure 3: Relative intensity noise (RIN) spectral density of five different sources: $20 \mathrm{~mW}$ (orange: 1) and $40 \mathrm{~mW}$ (red: 2) DBFs, IDPhotonics tunable laser (green: 3), RIO semiconductor external cavity laser - free running (blue-green: 4), Koheras fiber laser (blue: 5)

\section{DISCUSSION \& PERSPECTIVES}

In some applications, laser AM noise and FM noise can be both a problem for the overall system performance. This is the case of optoelectronic oscillators, where the optical AM noise is converted into RF phase noise at the photodiode level and the optical FM noise is converted into AM noise through dispersion effects in the fiber delay line or the reference cavity. In optical frequency synthesis systems, a source with low AM and FM noise is also of high interest. In this application, the source should feature large frequency tunability bandwidth together with low AM and FM noise. However, it is clear from the measurements depicted in Figures 2 and 3 that a source featuring high performance in all these parameters is hard to find. Lowering the laser frequency noise involves the stabilization of the source on an high $\mathrm{Q}$ resonator and this process often results in increased amplitude noise.

Nevertheless, a careful selection of a medium power or high power semiconductor laser can help to reduce both AM noise and FM noise. Laser 2 in this study would be a good 
choice for these applications. Therefore, the next step will be to try to stabilize this laser on an ultra-high $\mathrm{Q}$ resonator. The intrinsic low noise properties of this device, together with its high tunability should result in a high quality optical source with numerous potential applications.

\section{REFERENCES}

[1] D. Derickson, Fiber Optic Test and Measurement, Prentice Hall 1998.

[2] S. Camatel, V. Ferrero, "Narrow linewidth $\mathrm{CW}$ laser phase noise characterization methods for coherent transmission system applications", Journal of Lightwave Tech., vol 26, №17, Sept. 2008, pp. 3048-3055.

[3] O. Llopis, Z. Abdallah, V. Auroux, A. Fernandez, "High spectral purity laser characterization with a self-heterodyne frequency discriminator," Proc. of the IEEE Int. Freq. Control Symp. \& EFTF, Denver, CO, 2015, pp. 602-605.

[4] G. Bailly, O. Llopis and A. Fernandez, "High Spectral Purity Optical Source Stabilized on a Fiber Ring Resonator," IEEE Photonics Technology Letters, vol. 32, no. 5, pp. 255-258, March 2020. 